\title{
3D Sound Characterisation in Theatres Employing Microphone Arrays
}

\author{
Angelo Farina ${ }^{1)}$, Lamberto Tronchin ${ }^{2)}$ \\ 1) IED, University of Parma, Parma, Italy. farina@unipr.it \\ 1) DIENCA - CIARM, University of Bologna, Bologna, Italy. lamberto.tronchin@unibo.it
}

\begin{abstract}
Summary
The analysis of the sound field's 3D properties has been strongly improved in recent years, after spatial properties of sound propagation have been acknowledged to be important during the design or correction of theatres and auditoria. Besides, a proper assessment of spatial accuracy is requested for 3D sound reproduction systems, initially designed for acoustical virtual reality and now also employed in the entertainment/cinema industry (Immsound, Auro-3D, NHK 22.2). Often only monoaural or binaural measurements are performed by means of omni-directional microphones and dummy heads, although international standards like ISO 3382/1:2009 also define some "truly spatial" parameters such as JLF and JLFC. The two latter parameters are derived from measurements made with a pressure velocity (p/v) microphone, but this is still a 2-channel measurement. 3D Impulse Responses (4-channel B-format) have for many years been measured and employed for sound reproduction. Recently, higher-order 3D Impulse Responses have been measurable thanks to the availability of compact microphone arrays employing a much larger number of transducers. In this paper, two procedures for measuring and analysing the complete spatial sound information are presented, which are aimed at creating easy-to-understand images and videos showing the direction-of-arrival of the room reflections. The description of these techniques is emphasised. Furthermore, results are illustrated of experiments conducted with the novel methodologies in different rooms, including some ancient theatres.
\end{abstract}

PACS no. 43.55.Br, 43.55.Gx, 43.55.Mc, 43.60.Fg, 43.60.Lq, 43.60.Sx

\section{Introduction}

The need for capturing the acoustic properties and the sonic behaviour of significant ancient theatres and auditoria, as was initially proposed by Gerzon [1], became even more important after the destruction by fire of two theatres in Italy - the Teatro Petruzzelli, Bari and Teatro la Fenice, Venice [2].

Following these catastrophes many attempts were made to standardise the acoustic measurements in theatres and sacred spaces [3, 4] by taking into account several sound source positions, microphone positions and room conditions. On the other hand, only a few attempts were made to analyse and standardise the effect of test signals employed during the measurements and the types of sound source and microphones [5]. These details become crucial when measured Impulse Responses (IRs) are employed for performing $3 \mathrm{D}$ auralisation of the room, rather than for simply obtaining the numerical values of ISO 3382 parameters. Moreover, the full spatial sonic behaviour of a theatre, which includes information about energy, intensity and location of early reflections in the room, is required to deter-

Received 1 May 2012,

accepted 7 December 2012. mine and solve some acoustic problems that could not be resolved by only considering mono or 2-channel IRs.

\section{Previous measurement methods}

When Michael Gerzon first proposed measuring 3D Impulse Responses (with his Soundfield microphone probe, providing 4-channel B-format IRs) of ancient theatres and concert halls in order to assess and preserve their acoustical behaviour, he gathered only a few followers [1]. Only as of the new millennium, an international group of researchers agreed on the experimental methodology for collecting these measurements.

In order to obtain a complete description of spatial sound propagation in the auditorium, an updated measurement method was described in 2003 and 2005 [5, 6] which incorporates all the previously known measurement techniques in a single, coherent approach. Three different microphone systems were mounted on a rotating beam (a binaural dummy head, a pair of cardioids in ORTF configuration, and a Soundfield microphone), as shown if Figure 1, and a set of Impulse Responses were measured at each angular position. The ORTF configuration represents a standard method (adopted by French Radio/Television) for recording dual-channel signals, employing two cardioid microphones spaced $170 \mathrm{~mm}$ and divergent from 


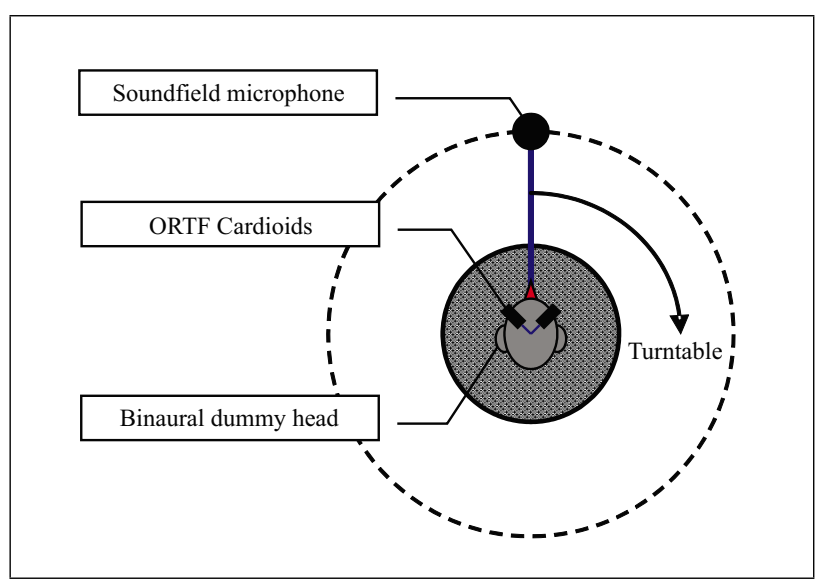

Figure 1. Measurement method previously proposed in 2005.

each other by 110 degrees. The Soundfield microphone, introduced by Gerzon, enabled the measurement of 4channel Impulse Responses and, therefore, spatial properties of the sound field. A Soundfield microphone captures a set of four signals known as "B-format" signals: one omnidirectional (sound pressure) and three with a polar pattern called "figure-of-eight", oriented along the three Cartesian axes X, Y, Z (these three channels capture a signal proportional to the Cartesian components of the particle velocity vector).

The combination of the three aforementioned different measurement methods provided a general method from which all standard multi-channel playback formats (i.e., 2.0, 5.1, 7.1, 10.2, etc.) could be derived.

\section{Enhanced methods for measuring multi- channel Impulse Responses}

The main problems with the method presented in $[5,6]$ were that it was very slow, the setup of the microphone system was tediously delicate and it was difficult to combine the information coming from the three microphone systems employed together. Recently, a much more powerful, elegant and simple recording/measurement system was proposed [7], based on a spherical microphone array equipped with 32 capsules mounted on the surface of a small sphere ( $80 \mathrm{~mm}$ diameter), containing the preamplifiers, A/D converters and an audio-over-ethernet chipset the Eigenmike ${ }^{\mathrm{TM}}$. This probe makes it possible to measure 3D multichannel Impulse Responses and provides much finer spatial resolution than previously possible.

\section{Test signal and sound source}

Techniques employed for the measurement of the Impulse Response have been significantly improved in recent years. The ESS (Exponential Sine Sweep) method [8] allows for the calculation of the Impulse Response of an electro-acoustical system by avoiding contamination due to distortions, which usually occur in the loudspeaker and provides a large value of the $\mathrm{S} / \mathrm{N}$ ratio. The choice of the sound source represents an important issue during the measurements and especially during the subsequent 3D auralisation. The ISO 3382/2009 standard [9] requires employing an omnidirectional sound source for measurements of room Impulse Responses.

The main advantage of the ESS signal method is that it provides a huge dynamic range (often in excess of $100 \mathrm{~dB}$ ) and is completely immune from nonlinear effects. The main problem, however, is that the signal must be radiated by a loudspeaker system. Even by employing a state-of-the-art, measurement grade dodecahedron loudspeaker, the source is never really an "omnidirectional point source". The polar pattern at medium-high frequencies deviates significantly from omnidirectionality, albeit the directivity limits of ISO 3382 are met. Furthermore, the radiated spectrum significantly changes with the direction and even with the distance from the sound source. Thus, for the analysis of spatial properties of rooms, it is better to employ sound sources which provide better omnidirectionality and a spatially-invariant spectrum such as specially-modified starter pistols equipped with an omnidirectional diffuser, or industrial-grade firecrackers, even if these sound sources provide smaller dynamic range (although usually better than $80 \mathrm{~dB}$ ) and a not-perfectly-flat spectrum [10].

Even though an omnidirectional source does not correspond to the effective directivity pattern of real-world sound sources, it is preferable when the purpose of the measurements is to precisely determine the spatial sound distribution in a room. It avoids exploiting room effects (abnormal concentration of energy and focusing for selected orientations of a directive source), as can happen when employing highly directive loudspeakers. However, when the purpose of the measurements is to determine the acoustic response of a room when a particular kind of source is used (a particular musical instrument or the human voice), a directive sound source could be added to the omnidirectional during the measurements (for example, an artificial mouth).

When employing an electrical test signal, the source is usually a dodecahedron (containing twelve loudspeakers) or another type of spherical source having a higher number of drivers. For wide-band Impulse Response measurements to be used for auralisation it is necessary to use a specially- built dodecahedron with wide frequency response $(30 \mathrm{~Hz}$ to $16 \mathrm{kHz}$, minimum) and incorporating a perfectly-flat digital equalisation system.

In many cases the need to employ a portable and cheap system makes it preferable to using "truly impulsive" sound sources such as starter pistols, balloons or firecrackers [10]. Recent studies [11] analysed the properties of balloons and revealed that they are, in general, unsatisfactory sound sources. Our own experiments, presently unpublished, reveal that industrial-grade firecrackers provide very good omnidirectionality, repeatability and a reasonably flat spectrum (unchanging with direction and distance, and hence easily perfectly flattened in post-processing). Industrial-grade firecrackers are there- 
fore the preferred sound source whenever a high quality dodecahedron loudspeaker cannot be used, or when omnidirectionality is crucial above the dynamic range or the flatness of the sound power level spectrum.

\section{Microphones}

So far, the ISO 3382/2009 standard [9] requires omnidirectional, monoaural microphones to be utilised in the measurement of monophonic acoustic parameters and only specifies the dimension of the microphones (preferably less than 13mm). Moreover, the ISO 3382/2009 standard describes the characteristics of binaural microphones (real heads or dummy heads), which can be used to measure binaural Impulse Responses and IACC. The standard also considers using figure-of-eight microphones to measure some lateral-energy parameters, such as LF and LFC, but does not provide technical specifications for these types of directive microphones. However, it is evident that monoaural or even figure-of-eight microphones cannot provide complete information about spatial sound distribution in the theatre. For this purpose, a multi-microphone system is necessary to capture the complete spatial information.

\subsection{B-format microphones}

Leaving aside binaural measurements, required only for binaural parameters, a B-format microphone (such as the Soundfield ${ }^{\mathrm{TM}}$ ) has been considered for many years to be the optimal transducer for performing 3D Impulse Response measurements in theatres and auditoria. The $\mathrm{W}$ channel is good for the monoaural parameters (omnidirectional), the $\mathrm{Y}$ channel provides the figure-of-eight signal required for computing LF, and the other two directive channels ( $\mathrm{X}$ and $\mathrm{Z}$ ) can be used for recreating the entire 3D soundscape inside a playback environment using the well-known 1st-order Ambisonics technology.

However, a 1st order Ambisonics playback system is currently considered incapable of providing accurate spatial cues to the listeners, as this technology does not synthesise sound fields exhibiting significant polarisation and consequently the sound is perceived to be coming from almost anywhere. A possible solution is to employ highorder Ambisonics (HOA) systems, which require capturing a multichannel signal corresponding to the spherical harmonics expansion up to 3rd or 4th orders. While it was found that HOA works very well with synthetic signals (where the high-order spherical harmonic signals are computer-generated), the recording of HOA signals is problematic, even when employing microphone arrays composed of dozens of elements - when the directivity of the harmonic pattern is high, the $\mathrm{S} / \mathrm{N}$ ratio is poor at low frequencies and the spatial accuracy of the pattern is disrupted at high frequencies, resulting in the reduction of the useful bandwidth to less than one octave band.

Another viable approach is to employ "advanced" decoding methods applied to the 1st order B-format signal, which perform a "spatial analysis" of the signal, and therefore "steer" the sound just in the very precise directions from where it arrives from, at every instant and at every frequency. Two of these methods are currently being employed, namely Sirr/Dirac by Pullki [12] and Harpex by Berge and Barrett [13]. The former is based on the Sound Intensity theory while the latter is based on plane wave decomposition.

\subsection{Large-number microphone arrays}

Recently, a 32-capsule microphone system was made available (Eigenmike ${ }^{\mathrm{TM}}$ ) and the authors developed a novel processing method of the signals captured by this probe, making it possible to directly synthesise a number of "virtual microphones" with arbitrary directivity pattern and aiming, without passing through HOA, spherical harmonics and the like [14].

\section{Spatial analysis of 3D Impulse Re- sponses}

Two 3D spatial analysis techniques were developed with the aim of graphically displaying the spatial sound distribution in the room. The first system was developed for processing 32-channel IRs measured by means of the Eigenmike $^{\mathrm{TM}}$ spherical microphone array [7]; the second was developed for analysing B-Format Impulse Response measured by means of a B-format microphone and is presented here for the first time.

\subsection{Spatial Analysis with the 3DVMS method}

For real-time recording/broadcasting applications, a very versatile microphone system was recently developed by the RAI Research Centre in Turin and by AIDA, a spinoff of the University of Parma [14]. The system is called 3DVMS (Three-Dimensional Virtual Microphone System) and is based on a 32-capsule spherical microphone array (Eigenmike ${ }^{\mathrm{TM}}$ ), along with a real-time filtering software which is capable of synthesising a number of virtual microphones, which can be moved in real-time and with variable directivity (zooming) capabilities.

The pattern is chosen among a family of cardioid microphones of various orders according to this formula,

$$
Q_{n}(\vartheta, \varphi)=[0.5+0.5 \cos (\vartheta) \cos (\varphi)]^{n},
$$

where $n$ is the directivity order of the microphone. These polar patterns are shown in Figure 2.

When performing the spatial analysis of a 32-channel Impulse Response, there is no need to move these virtual microphones. Instead, it is preferable to employ a set of 32 fixed virtual microphones, with 4th-order-cardioid fixed directivity patterns, pointing in the same directions as the capsules of the Eigenmike ${ }^{\mathrm{TM}}$, as shown in Figure 3.

For creating the signals of these 32 virtual microphones, a static matrix of $32 \times 32$ FIR filters is employed, thanks to a multichannel convolution tool [15]. In this way, the 


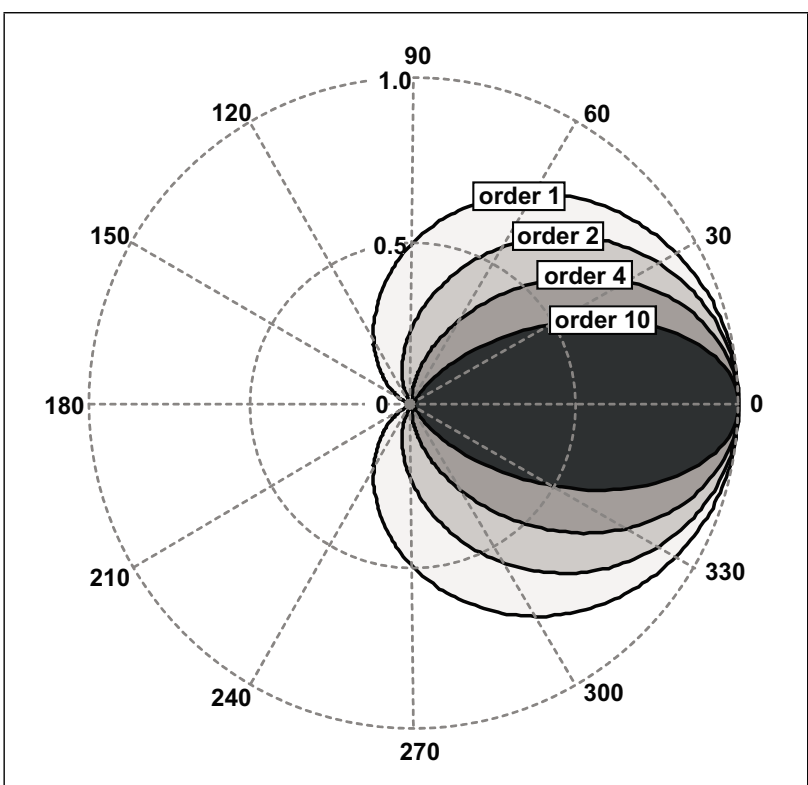

Figure 2. Directivity patterns of the virtual microphone.

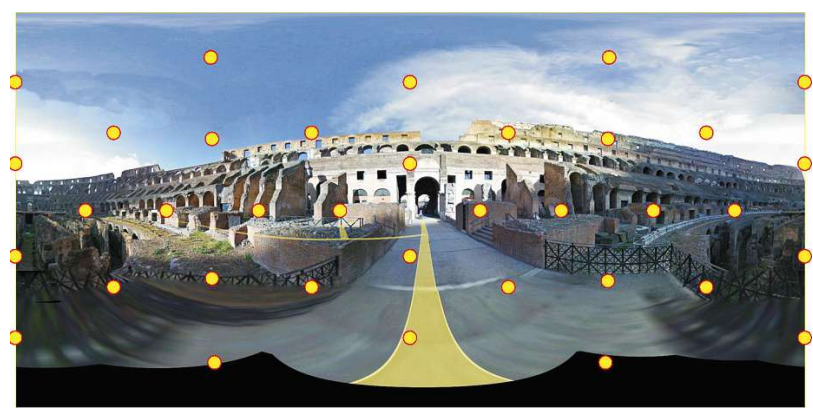

Figure 3 . The 32 virtual microphones pointing all around inside the Colosseum in Rome.

whole spherical surface is "spatially sampled" with an almost constant solid angular aperture. The resulting spatial sampling can therefore be considered as a "spatial PCM sampling", while the traditional spherical harmonics expansion employed in HOA can be thought as a "spatial Fourier sampling".

The results of the processing are plotted over a panoramic image taken from the microphone position, showing how much sound is coming from each direction. Hence, employing a standard graphical interpolation algorithm, it is possible to display a continuous colour map and this makes it possible to resolve reflections arriving simultaneously from different directions [7]. This colour map is recomputed for each $1 \mathrm{~ms}$-long time slice of the measured Impulse Response: the sequence of these images creates a sort of slow-motion video, showing how the spatial distribution of the sound arriving at the microphone changes over time.

This method can display detailed spatial information about early reflections in a theatre, although requiring the usage of expensive instrumentation. Furthermore, each Eigenmike probe requires its own set of "virtual microphone" FIR filters, so the raw 32-channel IRs measured with an Eigenmike cannot be considered a "standard" format. However, after processing through the $32 \times 32$ filter matrix, the resulting 32-channel Impulse Response becomes a new, standardised representation of the spatial information, which is called a spatially-sampled PCM signal [15], or P-format, and is independent from the particular microphone probe employed during the measurement, exactly as a B-format signal is independent from the particular tetrahedral microphone array employed.

\subsection{Spatial analysis from B-Format signals}

The second method exploits the capabilities of the Bformat signal of detecting the direction-of-arrival of each impinging wavefront by computing the "instantaneous" sound intensity vector I and the instantaneous value of the energy ratio $r_{E}$ and is based on the same vector decomposition scheme initially proposed in [16], also related to the later SIRR method [12]. The three components of the sound intensity vector can be simply obtained from the BFormat Impulse Response by means of the following equations:

$$
I_{x}=w \cdot x, \quad I_{y}=w \cdot y, \quad I_{z}=w \cdot z,
$$

where $w, x, y$ and $z$ represent the four signals of the Bformat IR. Keeping in mind these signals are proportional to sound pressure and particle velocity, the total energy density can be computed by means of

$$
E_{D}=\frac{w^{2}+x^{2}+y^{2}+z^{2}}{c} .
$$

It is also useful to compute the modulus of the sound intensity vector,

$$
|I|=\sqrt{I_{x}^{2}+I_{y}^{2}+I_{z}^{2}}
$$

The ratio between the active intensity and energy density is computed with

$$
r_{D}=\frac{|I|}{E_{D} \cdot c}=\frac{\sqrt{(w \cdot x)^{2}+(w \cdot y)^{2}+(w \cdot z)^{2}}}{w^{2}+x^{2}+y^{2}+z^{2}} .
$$

Finally, the azimuth (horizontal) $a$ and elevation (vertical) $e$ angles are simply obtained from trigonometric equations,

$$
a=\arctan \frac{I_{y}}{I_{x}}, \quad e=\arcsin \frac{I_{z}}{|I|} .
$$

All these quantities are averaged over $1 \mathrm{~ms}$ time slices, creating a "time history" of the above-defined descriptors along the whole length of the measured Impulse Response. The results can be visualised dynamically by means of a properly developed post-processing tool, plotting at every "frame" a circle, located at position $(a, e)$, having a diameter proportional to the sound intensity modulus $|I|$ and opacity proportional to $r_{E}$. The moving circle is plotted over a panoramic $360^{\circ} \times 180^{\circ}$ photographic image taken from the microphone position, while a synchronised marker moves over the Impulse Response graph so that 


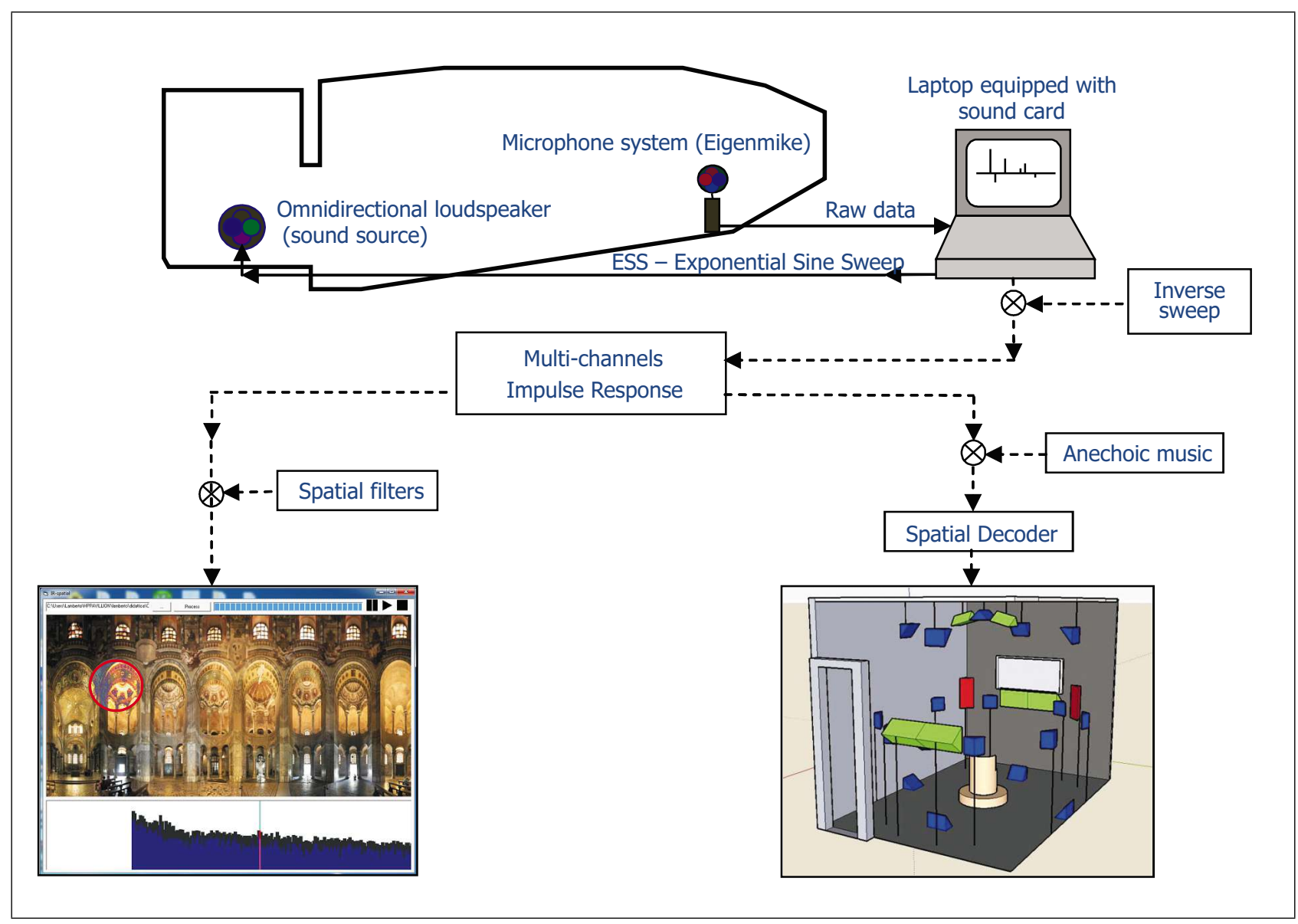

Figure 4. Flow chart of the measurement/analysis/playback method.

it is easy to see the arrival direction of each reflection and how much the corresponding wavefront is "polarised". The meaning of $r_{E}$ is related to the fact that the sound energy is significantly oriented along one direction $\left(r_{E}\right.$ approaching 1, travelling wave), or instead is diffuse ( $r_{E}$ approaching 0 , standing wave).

The chart does not display the "Impulse Response of $r_{E}$ ", but rather the superposition of the "energetic" Impulse Response (that is $E_{D}$ in $\mathrm{dB}$ ) and the "intensimetric" Impulse Response (that is $|I|$ in $\mathrm{dB}$ ), having aligned both $\mathrm{dB}$ scales so that, for a perfectly plane, progressive wave (when $r_{E}=1$ ) the two values in $\mathrm{dB}$ are the same for both $E_{D}$ and $|I|$.

Hence, the dynamic display of the spatial-temporal distribution of sound along the duration of the Impulse Response does not only carry the information of the trajectory of the reflected sound, but also about the degree of diffusion. The sound is fully diffused when the level of $E_{D}$ is much larger than the level of $|I|$ (and hence $r_{E}$ approaches 0 ). When, instead, the two levels are almost equal (meaning that $r_{E}=1$ ), the sound is strongly "polarised", a propagative wave traveling in a precise direction. This method allows for processing of a large amount of B-format IRs previously measured in the scientific community by means of Soundfield microphones or other tetrahedral probes, obtaining much more information than traditional acoustic parameters.

\section{Post Processing}

After measuring in each position a 3D Impulse Response (either B-format or 32-channel), it is possible to postprocess the results in two ways:

- A graphical analysis can be performed, showing the spatial distribution of the incoming energy along the running time; this allows us to "see" from where the room's reflections are coming

- An audible rendering can be presented to a group of listeners, inside a special room equipped with a suitable array of loudspeaker, surrounding completely the listening area within a sphere

The graphical analysis is performed thanks to two postprocessing software tools. The first shows the "moving circle", which corresponds to the instantaneous direction of arrival of the sound intensity, based on the analysis of a B-format Impulse Response performed according to the algorithm described in section 5.1. The second creates an animated colour video rendering of the sound map, plotted over the panoramic image. In this case no graphical algorithm is required, as a standard graphic library is employed for obtaining the colour map, based on the 32 "instantaneous" values of the sound pressure level captured by the 32 virtual microphones. These tools create an animated video rendering of the instantaneous sound intensity 


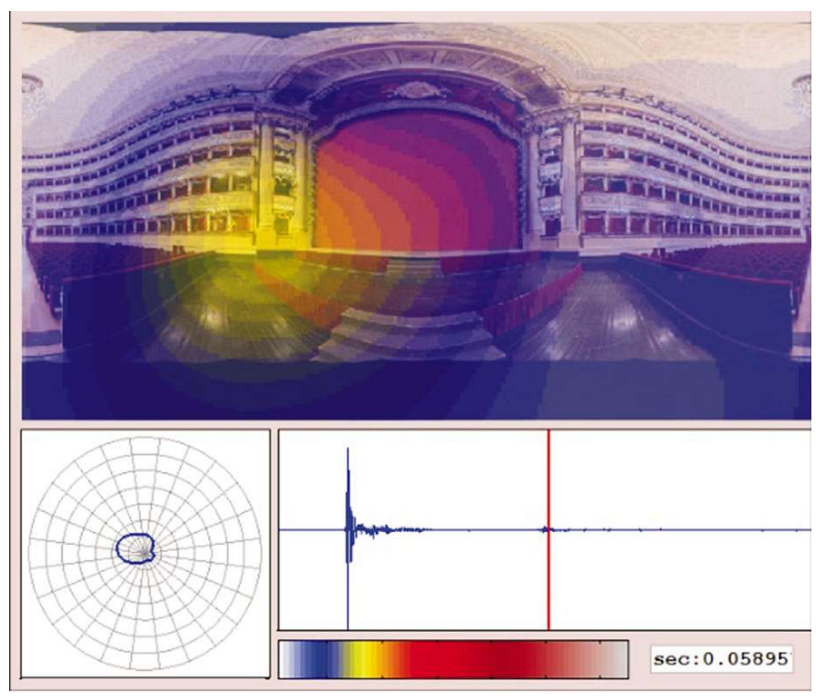

Figure 5. Snapshot of the video processing in La Scala Theater, a strong reflection from the side wall.

vector, in one case, and of a colour map of sound distribution, in the second case, plotted over the panoramic image. Frames of such a video rendering are shown in Figures 5-8.

The audible rendering is obtained by reprocessing the original Impulse Response recording - a new set of virtual microphones is extracted, one feeding each loudspeaker of the playback array. Again, the processing is slightly different for B-format Impulse Response, and for 32channel Impulse Responses, although the same methodological approach is employed. The set of filters employed for deriving the "playback" virtual microphones is obtained by solving a linear equation system, imposing that the signals re-recorded placing the probe (either the Bformat microphone or the Eigenmike ${ }^{\mathrm{TM}}$ ) at the centre of the playback system are maximally similar to the original signals recorded in the theatre. This approach, which is not Ambisonics-based, also corrects inherently for deviations from ideality of the loudspeakers employed, both in terms of magnitude/phase response, and in terms of placement/aiming/shielding.

\section{Flow chart}

A comprehensive flow chart of the "spatial" measurement/analysis/playback method is presented in Figure 4, showing how the signals are generated, captured, processed, analysed and reproduced.

\section{Experiments}

After testing the new measurement and post-processing systems in the Auditorium of Casa della Musica, Parma $[7,15]$, some experiments were conducted in a few historical rooms and theatres. The measurements presented here were conducted in different rooms and at different times and cover an historical Italian-style theatre, a modern Auditorium, an ancient Greek theatre and a sixth cen-

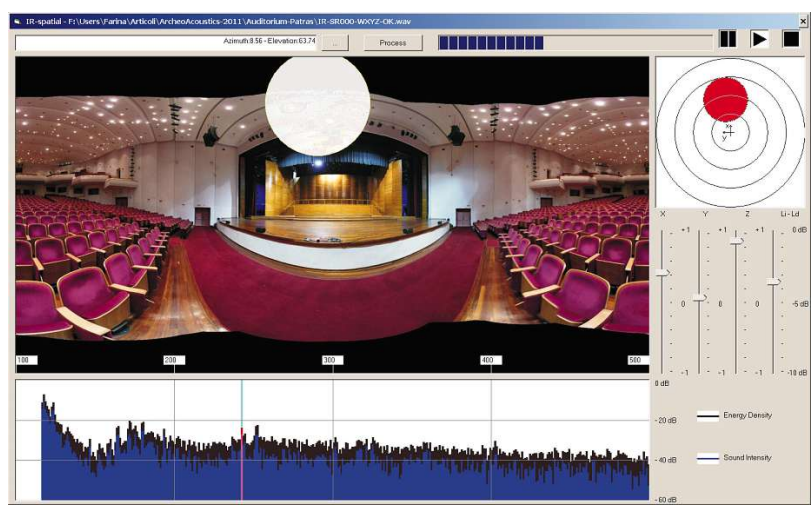

Figure 6. Snapshot of the video processing in the Auditorium of the University of Patras - Sound Intensity and Energy Density are highlighted.

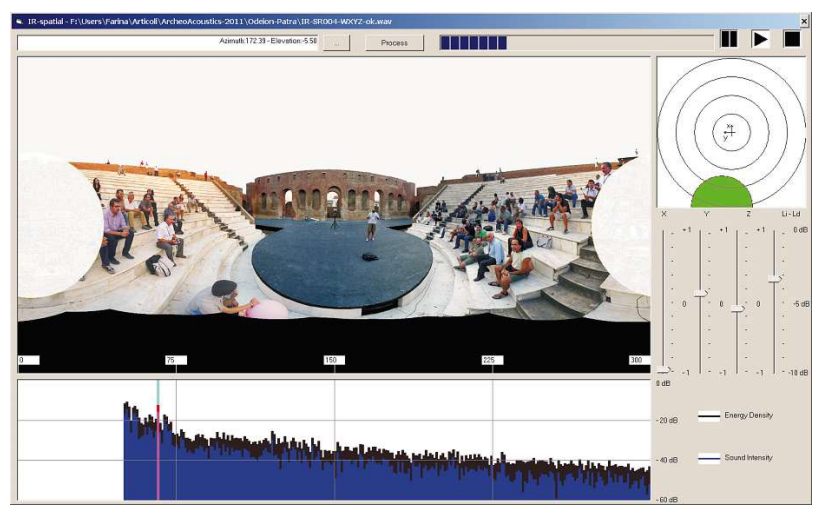

Figure 7. Snapshot of the video processing in Odeion theatre, Patras.

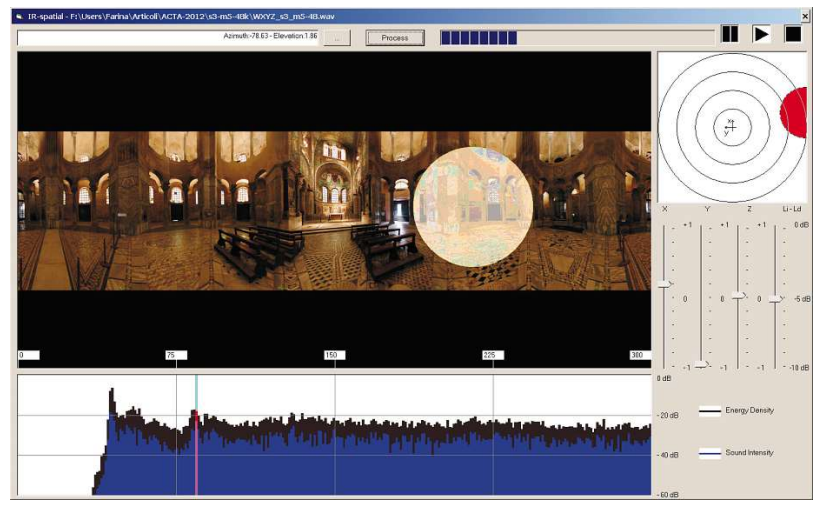

Figure 8. Snapshot of the video processing in San Vitale - A strong reflection from a lateral chapel.

tury Church. The novel processing methods create graphical animations that can be recorded in video files (AVI). In this paper, for each example we present figures containing a single frame of each of these videos (Figures $5,6,7,8)$. Most of the spatial information is understood by the acoustician by looking at these animations, as the temporal-spatial information appears with the evidence. Instead, looking at single frames taken at specific instants, the spatial analysis is much harder to perform. 
The four video files containing the analysis results of the following four cases are made available on the Internet [17]. It is suggested the reader watches these video files, rather than relying only on the single frames presented in the following sections.

\subsection{The Teatro alla Scala measurements}

The first example reported here was conducted inside the La Scala theatre in Milan. A set of 32-channel Impulse Responses was measured in ten positions along the stalls and boxes, using a measurement-grade and digitally equalised dodecahedron loudspeaker, fed with the ESS signal. Figure 5 shows a "frame" of the video rendering performed at the Director's position, showing a strong reflection bouncing back from the side wall. The 3D Impulse Responses measured at La Scala have also been employed for highquality spatial sound processing. The analysis performed with the 32-capsule array is also capable of discriminating multiple reflections arriving simultaneously to the microphone.

\subsection{The University of Patras Auditorium}

The second experiment was conducted inside the Auditorium of the University of Patras, during the Conference organised by the EAA in Patras, 2011. In this case, the Impulse Responses were measured in a few positions by means of a new A-Format microphone (Brahma ${ }^{\mathrm{TM}}$ ) [18]. Figure 6 shows a "frame" of the video rendering performed at the receiver's position in the third row. The conversion from A-Format to B-Format Impulse Response is obtained by means of a set of FIR filters.

The same mathematical framework described in [14] is employed. The Brahma probe is first characterised by means of a number of anechoic IR measurements from many directions. Then this matrix of measured IRs is inverted, creating a set of FIR filters (a matrix of $4 \times 4=16$ filters), which performs the conversion of the four "raw" capsule signals (A-format) into the four signals corresponding to the directivity patterns of four virtual microphones (B-format). This is exactly the same processing method described in [14] for the Eigenmike, but applied here to a probe equipped with just four microphones.

From the analysis of the processing results, shown in Figure 6, the effect of the proscenium on the Impulse Response is evident; it causes a strong late reflection that is detrimental to the acoustic quality of the room. By analysing these results, the solution to this echo problem consists of an acoustic treatment of the proscenium arch.

It must be noted how, in general, the use of B-format Impulse Responses only allows localising the direction of arrival of the early reflections, which are discrete and strongly polarised. After these, the sound field becomes more diffuse and the B-format method cannot properly analyse the spatial properties. This is easily seen from the difference between the levels of sound energy density and sound intensity, which becomes large. However, in this particular case, although this late reflection occurs more than $100 \mathrm{~ms}$ after the direct sound, the polarisation is still quite high, as shown from the fact that the difference between Energy Density and Sound Intensity levels is relatively small.

\subsection{The Odeion theatre in Patras}

The third experiment presented here was conducted in the Odeion theatre in Patras, Greece, also during the Conference organised by the EAA in Patras, 2011. In this case, the Impulse Responses were measured in a few positions by means of the same 4-channel microphone system described in the previous section and by using both a dodecahedron loudspeaker and firecrackers as sound sources. In this case, the latter worked better than the dodecahedron. Figure 7 shows an image from the video rendering performed at the receiver's position in the third row. The signals were post-processed and combined with the panoramic pictures of the theatre. Despite the low cost of the measuring system employed in this case, the use of firecrackers (which indeed did provide a high $\mathrm{S} / \mathrm{N}$ ratio) and of the 4-channel microphone system allowed for the proper reconstruction of the spatial sound distribution in a Greek theatre.

\subsection{The Basilica of San Vitale, Ravenna}

The last example represents the post-processing of measurements conducted in the sixth century Basilica of San Vitale in Ravenna in 2006 and presented in [19]. In this case, the B-Format Impulse Responses were measured by means of a Soundfield MKV microphone and of a digitally equalised dodecahedron sound source fed by the ESS signal. Taking into consideration the exact position of the microphone and of the sound source, the original measurements were post-processed and combined with a recent panoramic image of the church from the same microphone position, as shown in Figure 8.

In this case, the 4-channel method only allows for localising very early reflections, after which the value of $r_{E}$ becomes too small and the sound field becomes completely diffuse, making it meaningless to spatially analyse it. This is due to the fact that inside a church the energy carried by the reverberant sound field is very large. This example shows the possibility of obtaining spatial information about room acoustics from B-format measurements collected during past years, provided that the exact positions of sound source and microphone were accurately documented.

\section{Conclusions}

This article has described two novel methods for measuring and analysing 3D Impulse Responses in theatres, providing a spatial resolution significantly better than what was obtainable with existing technology (sum-anddelay beamforming for massive microphone arrays, 1st order Ambisonics for B-format microphones). Furthermore, these new methods produce easy-to-understand graphical animations of the spatial-temporal information. 
The first method is able to display very precise spatial information when a 32-channel spherical microphone array is used in the receiver position and is based on the synthesis of 32 highly-directive virtual microphones, which are obtained employing a huge matrix of FIR filters. However, useful results are obtainable also using 4-channel Bformat Impulse Responses employing the second method presented here, based on "instantaneous sound intensity" analysis. This makes it possible to analyse the spatial acoustical properties of theatres measured in the past by means of B-format microphones, provided that the exact position of sound source and microphone was documented and a panoramic photo was taken from each microphone position.

The system allows the acoustician to easily understand not only the acoustic behaviour of a room at a specific measuring position, but also to find out the direction from where the cause of a specific reflection is arriving. When the 32-channel microphone system is employed, it becomes possible to get a continuous colour map, showing the complete spatial distribution of the sound arriving at any given instant, by means of a number of highly directive virtual microphones, which operate a simultaneous spatial sampling of the whole sound field (a spatial filter bank). When a simpler B-format microphone probe is employed, the system currently can display just the instantaneous direction of the total energy flow, given by the sound intensity vector, accompanied by the information of the diffuseness of the sound field, given by the ratio $r_{E}$. However, the research is now assessing the possibility to reprocess a B-format Impulse Response with the available high resolution analysis methods (SIRR, Harpex), as these can, in principle, provide results similar to those currently obtained with the Eigenmike ${ }^{\mathrm{TM}}$ probe, at a fraction of the cost.

\section{Acknowledgement}

The Authors thank David J. Knight for his precious help during the proofread of the article.

\section{References}

[1] M. Gerzon: Recording concert hall acoustics for posterity. JAES 23 (1975) 569-571.

[2] L. Tronchin, A. Farina: The acoustics of the former teatro "La Fenice", Venice. JAES 45 (1997) 1051-1062.

[3] R. Pompoli, N. Prodi: Guidelines for acoustical measurements inside historical opera houses: procedures and validation. J.S.V. 232 (2000) 281-301.
[4] F. Martellotta, E. Cirillo, A. Carbonari, P. Ricciardi: Guidelines for acoustical measurements in churches. Applied Acoustics 70 (2009) 378-388.

[5] A. Farina, R. Ayalon: Recording concert hall acoustics for posterity. 24th AES Conference on Multichannel Audio, Banff, Canada, 26-28 June 2003.

[6] A. Farina, L. Tronchin: Measurement and reproduction of spatial sound characteristics of auditoria. Acoustical Science and Technology 26 (2005) 193-199.

[7] A. Farina, A. Amendola, A. Capra, C. Varani: Spatial analysis of room impulse responses captured with a 32-capsules microphone array. 130th AES Conference, London, 13-16 May 2011.

[8] A. Farina: Simultaneous measurement of impulse response and distortion with a swept-sine technique. 110th AES Convention, Paris, 18-22 February 2000.

[9] ISO 3382-1: Acoustics - Measurement of room acoustic parameters - Part 1: Performance spaces. 2009.

[10] A. Farina: Impulse response measurements. 23rd Nordic Sound Symposium, Bolkesjø, Norway, 27-30 September 2007.

[11] J. Pätynen, B. F. Katz, T. Lokki: Investigations on the balloon as an impulse source. J. Acoust. Soc. Am. 129 (2011) 27-33.

[12] V. Pulkki: Spatial sound reproduction with directional audio coding. J. Audio Eng. Soc. 55 (2007) 503-516.

[13] S. Berge, N. Barrett: High angular resolution planewave expansion. Proc. of the 2nd International Symposium on Ambisonics and Spherical Acoustics, Paris, France, May 6-7, 2010.

[14] A. Farina, A. Capra, L. Chiesi, L. Scopece: A spherical microphone array for synthesizing virtual directive microphones in live broadcasting and in post production. 40th AES Conference "Spatial Audio - Sense the Sound of Space, Tokyo, Japan, 8-10 October 2010.

[15] A. Farina, M. Binelli, A. Capra, E. Armelloni, S. Campanini, A. Amendola: Recording, simulation and reproduction of spatial soundfields by spatial PCM sampling (SPS). International Seminar on Virtual Acoustics, Valencia, Spain, 24-25 November 2011.

[16] A. Farina, E. Ugolotti: Subjective comparison between stereo dipole and 3D ambisonics surround systems for automotive applications. 16th AES Conference, Rovaniemi, Finland, 10-12 April 1999.

[17] Video files of the four examples: http://www.angelofarina.it/Public/AAA-2012.

[18] Brahma spatial sound recording system: http://www.aidasrl.it/brahma.html.

[19] L. Tronchin, D. J. Knight: The acoustical survival of San Vitale, Ravenna, Italy through two millennia. Proc. of Institute of Acoustics $\mathbf{3 0}$ (2008). 